\title{
A 25 micron search for Vega-like disks around main-sequence stars with ISO^
}

\author{
R. J. Laureijs ${ }^{1, \star \star}$, M. Jourdain de Muizon ${ }^{2,3, \star \star \star}$, K. Leech ${ }^{1, \dagger}$, R. Siebenmorgen ${ }^{4}$, C. Dominik ${ }^{5}$, \\ H. J. Habing ${ }^{6}$, N. Trams ${ }^{7}$, and M. F. Kessler ${ }^{1, \star \star}$ \\ 1 ISO Data Centre, ESA Astrophysics Division, Villafranca del Castillo, PO Box 50727, 28080 Madrid, Spain \\ 2 LAEFF-INTA, ESA VILSPA, PO Box 50727, 28080 Madrid, Spain \\ 3 DESPA, Observatoire de Paris, 92190 Meudon, France \\ 4 ESO, K. Schwarzschildstr. 2, 85748 Garching bei München, Germany \\ 5 Astr. Inst. Anton Pannekoek, Univ. Amsterdam, Kruislaan 403, 1098 SJ, Amsterdam, The Netherlands \\ ${ }^{6}$ Leiden Observatory, PO Box 9513, 2300 RA Leiden, The Netherlands \\ 7 Integral Science Operations, ESA ESTEC, Keplerlaan 1, 2201 AZ Noordwijk, The Netherlands
}

Received 10 August 2001 / Accepted 8 March 2002

\begin{abstract}
We present an ISO $25 \mu \mathrm{m}$ photometric survey of a sample of 81 nearby main-sequence stars in order to determine the incidence of "warm" dust disks. All stars were detected by ISO. We used an empirical relation to estimate the photospheric flux of the stars at $25 \mu \mathrm{m}$. We find 5 stars $(6 \%)$ with excess above the photospheric flux which we attribute to a Vega-like disk. These stars show disk temperatures not warmer than $120 \mathrm{~K}$. Our study indicates that warm disks are relatively rare. Not a single star in our sample older than 400 Myr has a warm disk. We find an upper limit of $M_{\text {disk }}=2 \times 10^{-5} M_{\oplus}$ for the mass of the disks which we did not detect.
\end{abstract}

Key words. stars: planetary systems - stars: general - infrared: stars

\section{Introduction}

After the initial discoveries by IRAS, the search and analysis of Vega-like disks in the infrared has received a substantial boost with the availability of data from the Infrared Space Observatory (ISO, Kessler et al. 1996). ISO has improved on IRAS in several important ways. Firstly, the number of bands has been increased and the infrared wavelength coverage has been extended to $200 \mu \mathrm{m}$; secondly, the detection limits have been lowered; and, thirdly, imaging and spectroscopy have been made possible on arcsec scales. One major ISO finding, based on a statistical study, is that the detection of a debris dust disk depends strongly on the age of a star: the probability of detecting a Vega-like disk comes close to unity for main-sequence dwarfs of less than 400 Myr (Habing et al. 1999; Habing et al. 2001, hereafter Paper I). Disks around older main-sequence stars are

Send offprint requests to: R. J. Laureijs,

e-mail: Rene.Laureijs@esa.int

* ISO is an ESA project with instruments funded by ESA Member States (especially the PI countries: France, Germany, The Netherlands and the United Kingdom) and with the participation of ISAS and NASA.

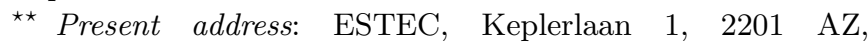
Noordwijk, The Netherlands

$\star \star \star$ Present address: Leiden Observatory

$\dagger$ Present address: Said Business School, Park End Street, Oxford, OX1 1HP, UK much less frequent, but they still exist. The precise mechanism that prevents these older disks from dissipating is still an open question (Jourdain de Muizon et al. 2001).

The search for new Vega-like stars has been based either on the analysis of the infrared colours using the IRAS database (Fajardo-Acosta et al. 2000; Mannings \& Barlow 1998, and references therein for previous IRAS surveys), or by comparing the far-infrared flux with a prediction based on a photospheric model or extrapolation from optical photometry. In most cases the surveys rely on the measurements at $60 \mu \mathrm{m}$, because the excess emission is high compared to the photospheric flux and the background confusion is low compared to observations at longer wavelengths.

A significant excess at shorter wavelengths is a signature of warm debris material, presumably closer to the star than the particles emitting at the longer wavelengths. Detecting such an excess generally requires a high photometric accuracy due to the relatively large contribution of the photospheric emission.

In this paper we analyse photometric data at $25 \mu \mathrm{m}$ of a sample of 81 main-sequence stars in order to determine the fraction of the stars which have significant infrared excess at $25 \mu \mathrm{m}$.

In Sect. 2 we describe the sample, the different data sets and the processing of the ISOPHOT data. In order to achieve the highest possible photometric accuracy we 
merged the ISO and IRAS data taking into account the systematic differences in calibration between the two data sets. The merging of the data sets and the extraction of the stars with $25 \mu \mathrm{m}$ excess are described in Sect. 3. The results are analysed in Sect. 4 . In Sect. 5 we discuss the properties of the excess stars. The conclusions are stated in Sect. 6.

\section{Observations and data processing}

\subsection{Presentation of the sample}

For a full description and discussion of the sample we refer to Paper I; here we briefly describe the properties of the $25 \mu \mathrm{m}$ sample. The stars were selected on the following selection criteria: (1) all stars are within $25 \mathrm{pc}$ of the Sun; (2) a spectral type later than B9 but earlier than M, and only dwarfs of type IV-V or V; (3) a predicted photospheric flux at $60 \mu \mathrm{m}$ of at least $30 \mathrm{mJy}$; (4) no (optical) binaries within the aperture; (5) no known variables.

The third selection criterion implies that the minimum photospheric flux at $25 \mu \mathrm{m}$ is of the order of $90 \mathrm{mJy}$. During the mission, some stars were added but these have not biased the statistics (cf. Paper I, Sect. 5.4).

In Table 1 we have listed the properties of the stars of the sample observed at $25 \mu \mathrm{m}$. Columns 1 to 3 describe the catalogue names of the stars, including the ISO observation identifier. Columns 4 to 6 list the optical stellar parameters from the Hipparcos Catalogue (Perryman et al. 1997), the spectral types in Col. 6 come from the machine-readable version of the Hipparcos Catalogue. Column 7 gives the age of the star from Lachaume et al. (1999). Columns 8 and 9 give the predicted flux density from Eq. (1) (below) and IRAS flux density, respectively. Columns 10 and 11 list flux density plus uncertainty obtained from the ISO data. Columns 12 and 13 give the adopted flux density plus uncertainty as described in Sect. 3.1. Finally, Col. 14 lists possible flags - "E" indicating a significant excess, and " $\mathrm{N}$ " indicating that the star was not included in the $60 \mu \mathrm{m}$ list of Paper I due to instrumental reasons and visibility constraints during the ISO mission.

\subsection{IRAS data}

The IRAS data were obtained from the IRAS faint source catalog (IFSC, Moshir et al. 1989). When no IFSC data are available we have taken data from the IRAS point source catalog.

\subsection{Predicted photospheric fluxes}

In order to decide whether a star has any excess emission at $25 \mu \mathrm{m}$ we have used the relation derived by Plets (1997):

$$
\begin{aligned}
V-[25]= & -0.03+2.99(B-V)-1.06(B-V)^{2} \\
& +0.47(B-V)^{3},
\end{aligned}
$$

which is based on the analysis of photometric data obtained in the optical and with IRAS of a very large sample of stars. The relation is valid for $B-V$ as high as 1.6 mag. However, investigation of the $V-[25]$ versus $B-V$ diagrams by Mathioudakis \& Doyle (1993) showed that K dwarfs may have higher infrared fluxes than predicted by Eq. (1) in case $B-V>1.2$ mag. We will take these findings into consideration when assessing stars with significant infrared excess.

Equation (1) is tied to the IRAS calibration. We adopted a flux density of $6.73 \mathrm{Jy}$ for [25] $=0 \mathrm{mag}$, this value is consistent with the IRAS photometric calibration (IRAS Explanatory Supplement).

For the analysis of likely excess stars we needed to estimate the photospheric flux at $12 \mu \mathrm{m}$. We used the relationship similar to Eq. (1) derived by Waters et al. (1987). The photospheric flux densities in the far-infrared $(\lambda \geq 60 \mu \mathrm{m})$ were estimated by assuming that for a given star the magnitude longward of $60 \mu \mathrm{m}$ is identical to the $60 \mu \mathrm{m}$ magnitude as given in Paper I.

The values of $V$ and $B-V$ were taken from the Hipparcos Catalogue (Perryman et al. 1997). At the low flux end with $F_{\nu}(25 \mu \mathrm{m})<300 \mathrm{mJy}$, an uncertainty of $m_{V}=0.01$ corresponds to about $2 \mathrm{mJy}$ and $B-V=0.01$ corresponds to about $5 \mathrm{mJy}$. Consequently, the statistical uncertainty in the "predicted" flux density according to Eq. (1) is estimated to be of the order of $2-3 \%$.

\subsection{ISO data processing}

The ISOPHOT data at $25 \mu \mathrm{m}$ (Lemke et al. 1996) were collected throughout the ISO mission (Leech \& Pollock 2000) with observation template AOT PHT03 in triangular chopped mode (Klaas et al. 1994). The chopper throw was $60^{\prime \prime}$ and the aperture used was $52^{\prime \prime}$. The on-target exposure time was $128 \mathrm{~s}$ and an equal amount of time was spent on the two background positions.

The data were processed using ISOPHOT interactive analysis PIA Version 8.1 (Gabriel et al. 1997). All standard signal corrections were applied. A generic chopper pattern of two plateaux of 4 "source plus background" and 4 "background" signals were derived. For the signal difference we have taken the average of the last two signals of each plateau. The signals were converted to flux densities under the assumption that the responsivity of the detector has the same value at the beginning of each ISO revolution, and changes with orbital phase due to ionising radiation according to an empirical function tabulated in the "Cal G" table PPRESP (Laureijs et al. 2001).

To check the ISO results we have correlated the ISO fluxes with the predicted fluxes and found a tight correlation. However, the correlation is not along the line of unit slope but along a power law where the high fluxes $\left(F_{\nu}>1 \mathrm{Jy}\right)$ are systematically underestimated and the lower fluxes $\left(F_{\nu}<300 \mathrm{mJy}\right)$ are systematically overestimated with respect to the model predictions, see Fig. 1. 
Table 1. The stars of the sample at $25 \mu \mathrm{m}$.

\begin{tabular}{|c|c|c|c|c|c|c|c|c|c|c|c|c|c|}
\hline HD & HIP & $\begin{array}{c}\text { ISO_id } \\
(3)\end{array}$ & $\begin{array}{c}V \\
\text { mag } \\
(4)\end{array}$ & $\begin{array}{c}B-V \\
\operatorname{mag} \\
(5)\end{array}$ & $\begin{array}{c}\text { Spect. } \\
\text { (6) }\end{array}$ & $\begin{array}{r}\text { age } \\
\text { Gyr } \\
(7)\end{array}$ & $\begin{array}{c}F_{\nu}^{\text {pred }} \\
\text { Jy } \\
(8)\end{array}$ & $\begin{array}{c}F_{\nu}^{\mathrm{IRAS}} \\
\mathrm{Jy} \\
(9)\end{array}$ & $\begin{array}{c}F_{\nu}^{\mathrm{ISO}} \\
\mathrm{Jy} \\
(10)\end{array}$ & $\begin{array}{c}\Delta F_{\nu}^{\mathrm{ISO}} \\
\mathrm{Jy} \\
(11)\end{array}$ & $\begin{array}{c}F_{\nu}^{\mathrm{ad}} \\
\mathrm{Jy} \\
(12)\end{array}$ & $\begin{array}{r}\Delta F_{\nu}^{\mathrm{ad}} \\
\mathrm{Jy} \\
(13)\end{array}$ & $\begin{array}{r}\text { Excess } \\
\text { Flag } \\
(14)\end{array}$ \\
\hline 693 & 910 & 37500901 & 4.89 & 0.487 & $\mathrm{~F} 5 \mathrm{~V}$ & 5.13 & 0.231 & 0.174 & 0.254 & 0.013 & 0.214 & 0.040 & \\
\hline 1581 & 1599 & 85900104 & 4.23 & 0.576 & F9V & 6.46 & 0.511 & 0.496 & 0.491 & 0.011 & 0.494 & 0.008 & \\
\hline 4628 & 3765 & 39502507 & 5.74 & 0.890 & K2V & 7.94 & 0.241 & & 0.209 & 0.016 & 0.209 & 0.016 & \\
\hline 4813 & 3909 & 38701510 & 5.17 & 0.514 & F7IV-V & 1.38 & 0.189 & 0.221 & 0.220 & 0.030 & 0.220 & 0.021 & \\
\hline 7570 & 5862 & 38603613 & 4.97 & 0.571 & F8V & 3.16 & 0.256 & 0.246 & 0.232 & 0.029 & 0.239 & 0.020 & \\
\hline 9826 & 7513 & 42301519 & 4.10 & 0.536 & $\mathrm{~F} 8 \mathrm{~V}$ & 2.88 & 0.530 & 0.519 & 0.532 & 0.021 & 0.526 & 0.015 & \\
\hline 10700 & 8102 & 39301216 & 3.49 & 0.727 & G8V & 7.24 & 1.373 & 1.542 & 1.096 & 0.023 & 1.319 & 0.223 & \\
\hline 10780 & 8362 & 45701319 & 5.63 & 0.804 & $\mathrm{~K} 0 \mathrm{~V}$ & 2.82 & 0.223 & 0.149 & 0.176 & 0.003 & 0.163 & 0.013 & \\
\hline 12311 & 9236 & 17902322 & 2.86 & 0.290 & F0V & 0.81 & 0.972 & 0.999 & 1.108 & 0.048 & 1.053 & 0.055 & \\
\hline 13445 & 10138 & 81301125 & 6.12 & 0.812 & K0V & 5.37 & 0.145 & 0.131 & 0.161 & 0.032 & 0.146 & 0.022 & \\
\hline 13709 & 10320 & 40101128 & 5.27 & -0.01 & $\mathrm{~A} 0 \mathrm{~V}$ & 0.34 & 0.049 & 0.112 & 0.076 & 0.026 & 0.094 & 0.018 & $\mathrm{~N}$ \\
\hline 14412 & 10798 & 40101731 & 6.33 & 0.724 & G8V & 7.24 & 0.100 & 0.107 & 0.151 & 0.016 & 0.129 & 0.022 & \\
\hline 14802 & 11072 & 40301534 & 5.19 & 0.608 & $\mathrm{G} 2 \mathrm{~V}$ & 5.37 & 0.225 & 0.244 & 0.266 & 0.039 & 0.255 & 0.028 & \\
\hline 15008 & 11001 & 15700537 & 4.08 & 0.034 & A3V & 0.45 & 0.168 & 0.174 & 0.138 & 0.001 & 0.156 & 0.018 & \\
\hline 17051 & 12653 & 41102840 & 5.40 & 0.561 & G3IV & 3.09 & 0.169 & 0.163 & 0.223 & 0.048 & 0.193 & 0.034 & \\
\hline 17925 & 13402 & 28302143 & 6.05 & 0.862 & $\mathrm{~K} 1 \mathrm{~V}$ & 0.08 & 0.171 & 0.189 & 0.202 & 0.023 & 0.196 & 0.016 & \\
\hline 19373 & 14632 & 81001846 & 4.05 & 0.595 & G0V & 3.39 & 0.627 & 0.600 & 0.674 & 0.018 & 0.637 & 0.037 & \\
\hline 20630 & 15457 & 79201552 & 4.84 & 0.681 & G5Vvar & 0.30 & 0.361 & 0.334 & 0.433 & 0.031 & 0.383 & 0.050 & \\
\hline 20766 & 15330 & 27506149 & 5.53 & 0.641 & G2V & 4.79 & 0.176 & 0.202 & 0.077 & 0.018 & 0.139 & 0.063 & \\
\hline 20807 & 15371 & 57801755 & 5.24 & 0.600 & G1V & 7.24 & 0.212 & 0.209 & 0.242 & 0.017 & 0.225 & 0.016 & \\
\hline 22001 & 16245 & 69100658 & 4.71 & 0.410 & F5IV-V & 2.04 & 0.231 & 0.242 & 0.251 & 0.012 & 0.247 & 0.008 & \\
\hline 22484 & 16852 & 79501561 & 4.29 & 0.575 & F9V & 5.25 & 0.482 & 0.486 & 0.536 & 0.036 & 0.511 & 0.025 & \\
\hline 26965 & 19849 & 84801864 & 4.43 & 0.820 & $\mathrm{~K} 1 \mathrm{~V}$ & 7.24 & 0.697 & 0.816 & 0.711 & 0.022 & 0.763 & 0.053 & \\
\hline 30495 & 22263 & 83901667 & 5.49 & 0.632 & G3V & 0.21 & 0.179 & 0.160 & 0.161 & 0.010 & 0.160 & 0.007 & \\
\hline 33262 & 23693 & 58900870 & 4.71 & 0.526 & F7V & 2.95 & 0.296 & 0.314 & 0.236 & 0.020 & 0.275 & 0.039 & \\
\hline 34411 & 24813 & 83801473 & 4.69 & 0.630 & G0V & 6.76 & 0.373 & 0.343 & 0.269 & 0.016 & 0.306 & 0.037 & \\
\hline 37394 & 26779 & 83801976 & 6.21 & 0.840 & $\mathrm{~K} 1 \mathrm{~V}$ & 0.34 & 0.141 & 0.146 & 0.156 & 0.006 & 0.151 & 0.005 & \\
\hline 38392 & & 70201401 & 6.15 & 0.940 & $\mathrm{~K} 2 \mathrm{~V}$ & 0.87 & 0.183 & 0.153 & 0.207 & 0.027 & 0.180 & 0.027 & \\
\hline 38393 & 27072 & 70201304 & 3.59 & 0.481 & F7V & 1.66 & 0.755 & 0.701 & 0.849 & 0.031 & 0.775 & 0.074 & \\
\hline 38678 & 27288 & 69202307 & 3.55 & 0.104 & A2Vann & 0.37 & 0.328 & 0.826 & 0.748 & 0.040 & 0.787 & 0.039 & $\mathrm{E}$ \\
\hline 39060 & 27321 & 70201079 & 3.85 & 0.171 & A3V & 0.28 & 0.294 & 6.294 & 5.810 & 0.205 & 6.052 & 0.242 & $\mathrm{E}$ \\
\hline 43834 & 29271 & 19000682 & 5.08 & 0.714 & G5V & 7.24 & 0.309 & 0.286 & 0.334 & 0.039 & 0.310 & 0.027 & \\
\hline 48915 & 32349 & 72301710 & -1.4 & 0.009 & $\mathrm{~A} 0 \mathrm{~m} . .$. & & 25.28 & 24.264 & 20.222 & 0.625 & 22.243 & 2.021 & \\
\hline 50281 & 32984 & 71802113 & 6.58 & 1.071 & K3V & 2.63 & 0.162 & & 0.185 & 0.029 & 0.185 & 0.029 & \\
\hline 74576 & 42808 & 15600188 & 6.58 & 0.917 & $\mathrm{~K} 2 \mathrm{~V}$ & 0.81 & 0.117 & 0.284 & 0.140 & 0.018 & 0.212 & 0.072 & $\mathrm{~N}$ \\
\hline 84737 & 48113 & 14300497 & 5.08 & 0.619 & $\mathrm{G} 2 \mathrm{~V}$ & 5.37 & 0.255 & 0.234 & 0.261 & 0.004 & 0.247 & 0.014 & $\mathrm{~N}$ \\
\hline 88230 & 49908 & 14500801 & 6.60 & 1.326 & $\mathrm{~K} 8 \mathrm{~V}$ & 7.59 & 0.285 & 0.418 & 0.407 & 0.007 & 0.412 & 0.005 & $\mathrm{E}, \mathrm{N}$ \\
\hline 90839 & 51459 & 13100204 & 4.82 & 0.541 & F8V & 1.18 & 0.276 & 0.279 & 0.392 & 0.094 & 0.335 & 0.067 & $\mathrm{~N}$ \\
\hline 95128 & 53721 & 18000207 & 5.03 & 0.624 & G0V & 6.31 & 0.270 & 0.222 & 0.236 & 0.023 & 0.229 & 0.016 & $\mathrm{~N}$ \\
\hline 97603 & 54872 & 16900610 & 2.56 & 0.128 & $\mathrm{~A} 4 \mathrm{~V}$ & 0.68 & 0.868 & & 0.894 & 0.021 & 0.894 & 0.021 & $\mathrm{~N}$ \\
\hline
\end{tabular}

Assuming that the majority of the stars in our sample have no significant excess emission at $25 \mu \mathrm{m}$ we recalibrated the ISO fluxes to the predicted fluxes so that the correlation between subsamples of the two data sets scatters around the line of unit slope. The method is illustrated in Fig. 1 where the correlations are given between the ISO and IRAS fluxes before and after the correction. A detailed description of the recalibration is given elsewhere (Laureijs \& Jourdain de Muizon 2000). It should be stressed that the recalibration systematically changes the fluxes in the ISO sample as a whole and does not affect the relative scatter amoung the individual observations.
Also, the Plets (1997) predictions are based on the IRAS calibration, whereas the ISOPHOT calibration is based on a different photometric system. The recalibration ensures that the fluxes of the ISO observations are consistent with the IRAS calibration.

\section{Results}

\subsection{Extraction of $25 \mu \mathrm{m}$ excess stars}

The predicted, IRAS, and recalibrated ISO fluxes are listed in Table 1. The IRAS and ISO fluxes have been colour corrected assuming a stellar photosphere, the 
Table 1. continued.

\begin{tabular}{|c|c|c|c|c|c|c|c|c|c|c|c|c|c|}
\hline HD & $\begin{array}{l}\text { HIP } \\
(2)\end{array}$ & $\begin{array}{c}\text { ISO_id } \\
(3)\end{array}$ & $\begin{array}{c}V \\
\operatorname{mag} \\
(4)\end{array}$ & $\begin{array}{c}B-V \\
\operatorname{mag} \\
(5)\end{array}$ & Spect. & $\begin{array}{r}\text { age } \\
\text { Gyr } \\
(7)\end{array}$ & $\begin{array}{c}F_{\nu}^{\text {pred }} \\
\text { Jy } \\
(8)\end{array}$ & $\begin{array}{c}F_{\nu}^{\mathrm{IRAS}} \\
\text { Jy } \\
(9)\end{array}$ & $\begin{array}{c}F_{\nu}^{\mathrm{ISO}} \\
\mathrm{Jy} \\
(10)\end{array}$ & $\begin{array}{c}\Delta F_{\nu}^{\mathrm{ISO}} \\
\mathrm{Jy} \\
(11)\end{array}$ & $\begin{array}{c}F_{\nu}^{\mathrm{ad}} \\
\mathrm{Jy} \\
(12)\end{array}$ & $\begin{array}{r}\Delta F_{\nu}^{\mathrm{ad}} \\
\mathrm{Jy} \\
(13)\end{array}$ & $\begin{array}{r}\text { Excess } \\
\text { Flag } \\
(14)\end{array}$ \\
\hline 101501 & 56997 & 17200316 & 5.31 & 0.723 & G8Vvar & 1.55 & 0.255 & & 0.308 & 0.027 & 0.308 & 0.027 & $\mathrm{~N}$ \\
\hline 102365 & 57443 & 25400519 & 4.89 & 0.664 & G3/G5V & 7.24 & 0.333 & 0.351 & 0.374 & 0.016 & 0.362 & 0.011 & $\mathrm{~N}$ \\
\hline 102647 & 57632 & 18401422 & 2.14 & 0.090 & A3Vvar & 0.24 & 1.160 & 1.659 & 1.467 & 0.055 & 1.563 & 0.096 & $\mathrm{E}$ \\
\hline 106591 & 59774 & 14301528 & 3.32 & 0.077 & A3Vvar & 0.48 & 0.378 & 0.386 & 0.422 & 0.016 & 0.404 & 0.018 & \\
\hline 110833 & 62145 & 60000525 & 7.01 & 0.936 & K3V & 12.60 & 0.082 & & 0.090 & 0.022 & 0.090 & 0.022 & \\
\hline 112758 & 63366 & 40100128 & 7.54 & 0.769 & $\mathrm{KOV}$ & 5.89 & 0.036 & & 0.075 & 0.036 & 0.075 & 0.036 & $\mathrm{~N}$ \\
\hline 114710 & 64394 & 21501031 & 4.23 & 0.572 & G0V & 3.63 & 0.507 & 0.461 & 0.436 & 0.041 & 0.449 & 0.029 & \\
\hline 114762 & 64426 & 39000531 & 7.30 & 0.525 & F9V & 11.22 & 0.027 & & 0.030 & 0.021 & 0.030 & 0.021 & $\mathrm{~N}$ \\
\hline 115383 & 64792 & 24101534 & 5.19 & 0.585 & G0Vs & 3.80 & 0.215 & 0.191 & 0.208 & 0.021 & 0.199 & 0.015 & $\mathrm{~N}$ \\
\hline 117176 & 65721 & 39600734 & 4.97 & 0.714 & G5V & 7.59 & 0.342 & 0.384 & 0.315 & 0.006 & 0.349 & 0.034 & $\mathrm{~N}$ \\
\hline 120136 & 67275 & 39400137 & 4.50 & 0.508 & F7V & 1.38 & 0.346 & 0.346 & 0.322 & 0.007 & 0.334 & 0.012 & $\mathrm{~N}$ \\
\hline 126660 & 70497 & 19501343 & 4.04 & 0.497 & F7V & 2.95 & 0.516 & 0.517 & 0.558 & 0.039 & 0.537 & 0.027 & \\
\hline 128167 & 71284 & 26900346 & 4.47 & 0.364 & F3Vwvar & 1.70 & 0.261 & 0.295 & 0.314 & 0.043 & 0.305 & 0.030 & \\
\hline 134083 & 73996 & 08901049 & 4.93 & 0.429 & F5V & 1.82 & 0.197 & 0.176 & 0.217 & 0.009 & 0.197 & 0.020 & \\
\hline 139664 & 76829 & 09000155 & 4.64 & 0.413 & F5IV-V & 1.12 & 0.248 & 0.493 & 0.253 & 0.032 & 0.373 & 0.120 & \\
\hline 142373 & 77760 & 28100658 & 4.60 & 0.563 & F9V & 8.51 & 0.354 & 0.408 & 0.388 & 0.035 & 0.398 & 0.025 & \\
\hline 142860 & 78072 & 08901261 & 3.85 & 0.478 & F6V & 3.24 & 0.591 & 0.630 & 0.763 & 0.031 & 0.697 & 0.067 & \\
\hline 149661 & 81300 & 80700364 & 5.77 & 0.827 & $\mathrm{~K} 2 \mathrm{~V}$ & 2.09 & 0.206 & 0.224 & 0.173 & 0.014 & 0.198 & 0.026 & \\
\hline 154088 & 83541 & 45801567 & 6.59 & 0.814 & $\mathrm{~K} 1 \mathrm{~V}$ & 7.24 & 0.094 & & 0.089 & 0.009 & 0.089 & 0.009 & \\
\hline 156026 & 84478 & 09401670 & 6.33 & 1.144 & $\mathrm{~K} 5 \mathrm{~V}$ & 0.63 & 0.239 & & 0.266 & 0.017 & 0.266 & 0.017 & \\
\hline 157214 & 84862 & 09101273 & 5.38 & 0.619 & G0V & 7.24 & 0.193 & 0.196 & 0.182 & 0.023 & 0.189 & 0.016 & \\
\hline 157881 & 85295 & 09202576 & 7.54 & 1.359 & $\mathrm{~K} 7 \mathrm{~V}$ & 5.25 & 0.130 & 0.112 & 0.185 & 0.022 & 0.148 & 0.036 & \\
\hline 160691 & 86796 & 45800282 & 5.12 & 0.694 & G5V & 6.17 & 0.286 & 0.321 & 0.244 & 0.036 & 0.282 & 0.038 & \\
\hline 166620 & 88972 & 36901485 & 6.38 & 0.876 & K2V & 7.24 & 0.130 & 0.133 & 0.136 & 0.011 & 0.135 & 0.007 & \\
\hline 172167 & 91262 & 08900788 & 0.03 & -0.00 & A0Vvar & 0.35 & 6.351 & 8.079 & 8.234 & 0.305 & 8.156 & 0.215 & $\mathrm{E}$ \\
\hline 173667 & 92043 & 10600291 & 4.19 & 0.483 & F6V & 2.40 & 0.436 & 0.430 & 0.445 & 0.011 & 0.437 & 0.008 & \\
\hline 185144 & 96100 & 28801094 & 4.67 & 0.786 & $\mathrm{KOV}$ & 5.50 & 0.522 & 0.553 & 0.554 & 0.017 & 0.553 & 0.012 & \\
\hline 185395 & 96441 & 54801897 & 4.49 & 0.395 & $\mathrm{~F} 4 \mathrm{~V}$ & 1.29 & 0.274 & 0.221 & 0.236 & 0.029 & 0.229 & 0.021 & \\
\hline 187642 & 97649 & 13001001 & 0.76 & 0.221 & A7IV-V & 1.23 & 5.723 & 5.757 & 5.166 & 0.179 & 5.462 & 0.296 & \\
\hline 191408 & 99461 & 34301404 & 5.32 & 0.868 & $\mathrm{~K} 2 \mathrm{~V}$ & 7.24 & 0.339 & 0.450 & 0.474 & 0.027 & 0.462 & 0.019 & $\mathrm{E}$ \\
\hline 192310 & 99825 & 18300407 & 5.73 & 0.878 & $\mathrm{~K} 3 \mathrm{~V}$ & & 0.237 & 0.289 & 0.311 & 0.025 & 0.300 & 0.018 & \\
\hline 197692 & 102485 & 13501110 & 4.13 & 0.426 & F5V & 2.00 & 0.408 & 0.367 & 0.387 & 0.034 & 0.377 & 0.024 & \\
\hline 203280 & 105199 & 08900313 & 2.45 & 0.257 & A7IV-V & 0.89 & 1.314 & 1.220 & 1.262 & 0.029 & 1.241 & 0.021 & \\
\hline 203608 & 105858 & 10902816 & 4.21 & 0.494 & F6V & 10.47 & 0.439 & 0.459 & 0.459 & 0.017 & 0.459 & 0.012 & \\
\hline 207129 & 107649 & 13500819 & 5.57 & 0.601 & $\mathrm{G} 2 \mathrm{~V}$ & 6.03 & 0.156 & 0.143 & 0.269 & 0.030 & 0.206 & 0.063 & \\
\hline 209100 & 108870 & 18300322 & 4.69 & 1.056 & $\mathrm{~K} 5 \mathrm{~V}$ & 1.29 & 0.894 & 0.980 & 1.155 & 0.048 & 1.067 & 0.087 & \\
\hline 215789 & 112623 & 14401525 & 3.49 & 0.083 & A3V & 0.54 & 0.328 & 0.354 & 0.384 & 0.032 & 0.369 & 0.023 & \\
\hline 216956 & 113368 & 35300828 & 1.17 & 0.145 & A3V & 0.22 & 3.259 & 3.419 & 3.414 & 0.057 & 3.417 & 0.041 & $\mathrm{E}$ \\
\hline 217014 & 113357 & 37401640 & 5.45 & 0.666 & G5V & 5.13 & 0.200 & 0.175 & 0.179 & 0.031 & 0.177 & 0.022 & \\
\hline 219134 & 114622 & 09102431 & 5.57 & 1.000 & K3Vvar & & 0.353 & 0.387 & 0.349 & 0.028 & 0.368 & 0.020 & \\
\hline 222368 & 116771 & 37800834 & 4.13 & 0.507 & F7V & 3.80 & 0.485 & 0.526 & 0.524 & 0.039 & 0.525 & 0.028 & \\
\hline
\end{tabular}

Notes: HD 106591 was observed twice, the ISO flux density is the weighted average; the second ISO_id is 33700128.

HD 110833 is not in the list of Lachaume et al. (1999), the age is estimated by us according to Lachaume et al. (1999)

colour correction factors are 1.40 and 1.28 for IRAS and ISO, respectively. The table includes the adopted flux $F_{\nu}^{\text {ad }}$ at $25 \mu \mathrm{m}$, determined in the following way:

$$
F_{\nu}^{\mathrm{ad}}=\left(F_{\nu}^{\mathrm{ISO}}+F_{\nu}^{\mathrm{IRAS}}\right) / 2
$$

$\Delta F_{\nu}^{\mathrm{ad}}=\max \left(\Delta F_{\nu}^{\mathrm{ISO}} / \sqrt{2},\left(F_{\nu}^{\mathrm{ISO}}-F_{\nu}^{\mathrm{IRAS}}\right) / 2\right)$.
We make the conservative assumption that for most of the individual measurements the uncertainty in the IRAS flux is as large as that of ISO. We checked the validity of this assumption by analysing the distribution of residual fluxes $F_{\nu}-F_{\nu}^{\text {pred }}$ for the IRAS and ISO measurements separately (cf. Fig. 3). We found that both distributions have similar FWHM, of about $80 \mathrm{mJy}$, which suggests similar 


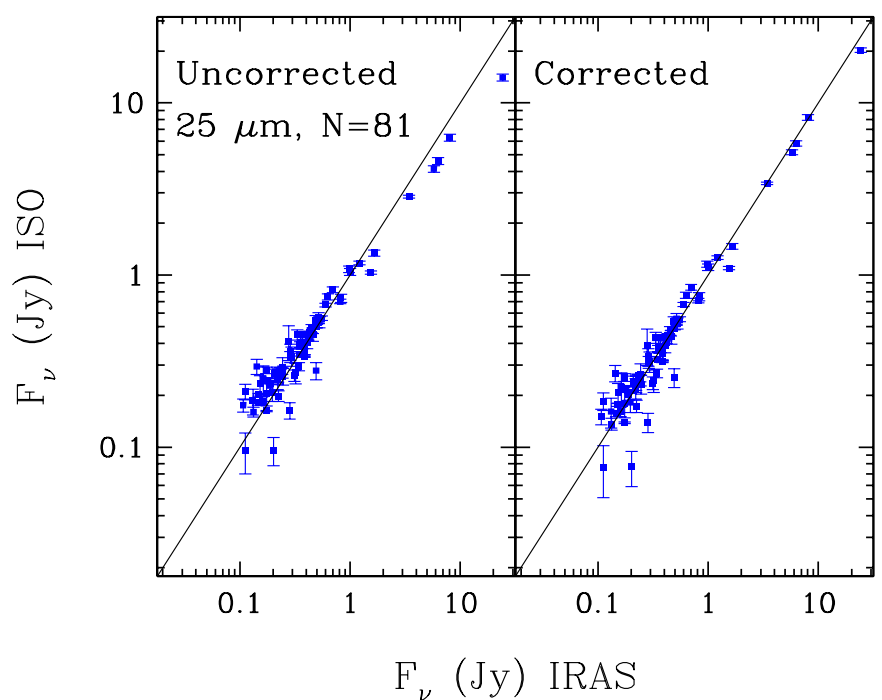

Fig. 1. Comparison between the photometric data of ISO and IRAS. Left panel: the correlation between ISO and IRAS. For the ISOPHOT data at $25 \mu \mathrm{m}$ we assumed a default detector responsivity and standard calibrations. Right panel: the same correlation after correction of the ISO fluxes to obtain an overall match with the predicted fluxes. The solid lines represent the lines of unit slope.

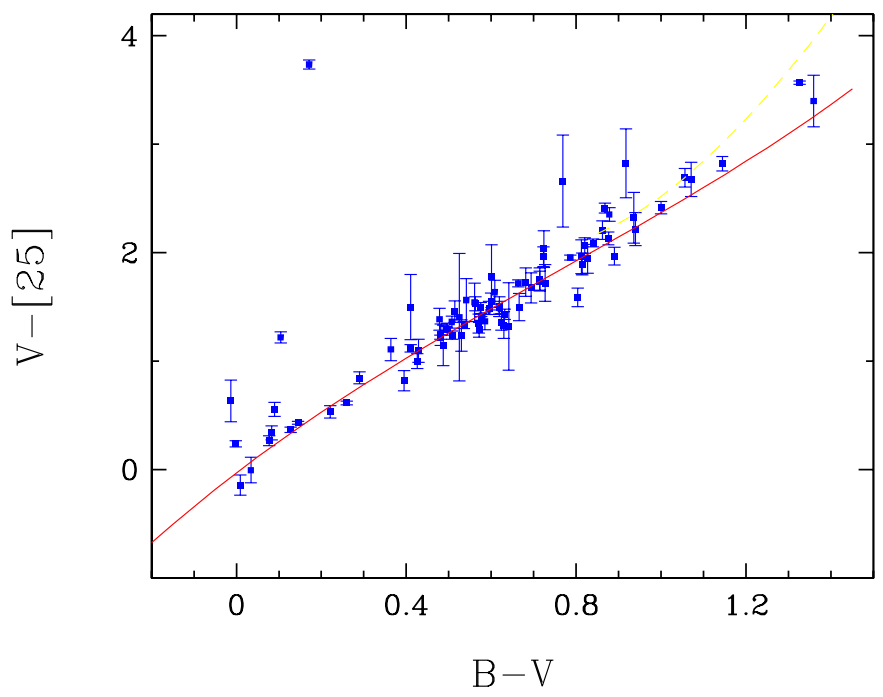

Fig. 2. $V-[25]$ versus $B-V$ colour for all stars in the sample. The solid line is the photospheric emission as predicted by Eq. (1). The dashed line is the relationship for $\mathrm{K}$ and $\mathrm{M}$ dwarfs with $B-V>0.8$ derived by Mathioudakis \& Doyle (1993).

uncertainties for the IRAS and ISO data. In case no IRAS flux is available the ISO flux and uncertainty were adopted.

The visual-infrared colour-colour diagram for the stars in the sample is presented in Fig. 2. The predicted photospheric flux follows closely the distribution of points in the sample indicating that Eq. (1) is applicable. The good match also indicates that most of the $25 \mu \mathrm{m}$ flux densities are predominantly photospheric. The sample contains 5 stars with $B-V>1.0$ (Table 1 ), these stars are all $\mathrm{K}$ dwarfs and have $V-[25]$ above the prediction. On the

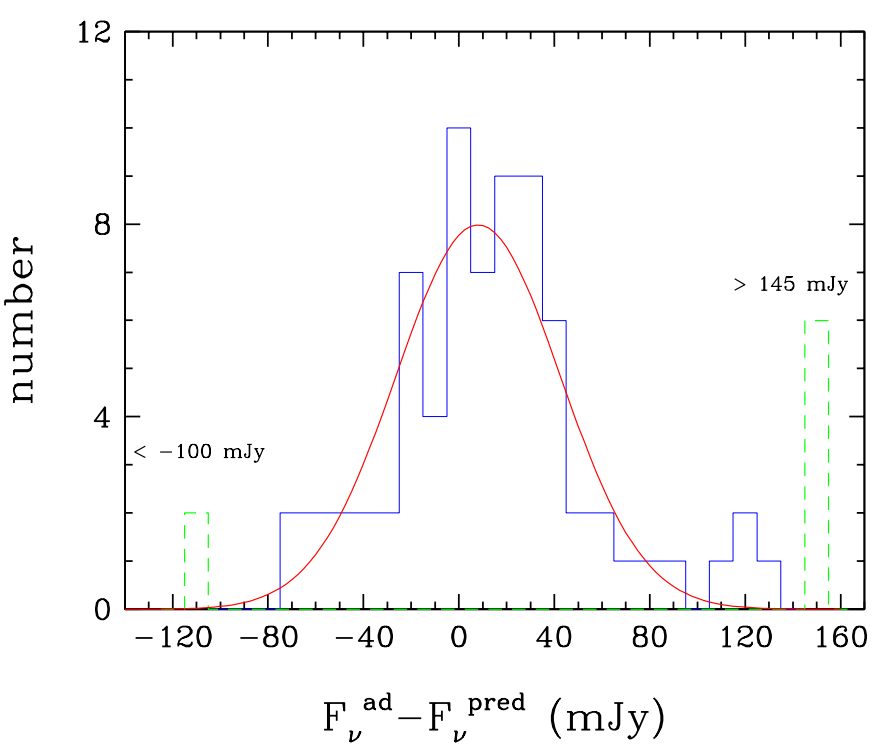

Fig. 3. Distribution of deviations from the predicted fluxes. The dashed bins give the number of stars below and above the given flux limits. The solid line is a normal distribution based on $\sigma=35 \mathrm{mJy}$, mean $=8 \mathrm{mJy}$.

other hand, the relation derived by Mathioudakis \& Doyle (1993) for $\mathrm{K}$ and $\mathrm{M}$ dwarfs predicts values of $V-[25]$ which are too high for the three stars with highest $V-[25]$.

To assess the photometric quality of the sample we present a histogram of the difference $F_{\nu}^{\text {ad }}-F_{\nu}^{\text {pred }}$ in Fig. 3. The distribution is strongly peaked and suggests a normal distribution close to zero for the majority of stars in the sample.

The parameters of the normal distribution have been derived as follows. Initially, an intermediate mean and standard deviation was derived of the stars in the interval $\left|F_{\nu}^{\text {ad }}-F_{\nu}^{\text {pred }}\right|<100$ mJy. Judging from the histogram we decided that the stars falling outside this interval must be outliers. Subsequently, all stars were rejected which are more than 2.6 standard deviations away from the mean (i.e. $\leq 1 \%$ probability of occurrence). This yields an interval of $-81 \mathrm{mJy}<F_{\nu}^{\text {ad }}-F_{\nu}^{\text {pred }}<97 \mathrm{mJy}$. The mean of the remaining stars is $8 \mathrm{mJy}$ with a dispersion of $35 \mathrm{mJy}$. The normal distribution has been included in Fig. 3. Application of a Kolmogorov-Smirnov test showed that the distribution in the given interval is normal with a significance level of $5 \%$.

This analysis indicates that for the majority of the stars in the sample, the $25 \mu \mathrm{m}$ fluxes are consistent with the expected photospheric fluxes. The overall scatter between the observations and expectations is $35 \mathrm{mJy}$. Judging from the individual uncertainties of the $25 \mu \mathrm{m}$ fluxes, we conclude that most of the scatter must come from the infrared measurements and that the predicted fluxes which are only based on optical data are very accurate, within $8 \mathrm{mJy}$ for the sample average. In addition, there is no indication that the distribution is nonnormal, suggesting that there is no statistical evidence for a surplus of positive excesses in the distribution for $F_{\nu}^{\text {ad }}-F_{\nu}^{\text {pred }}<97 \mathrm{mJy}$ 


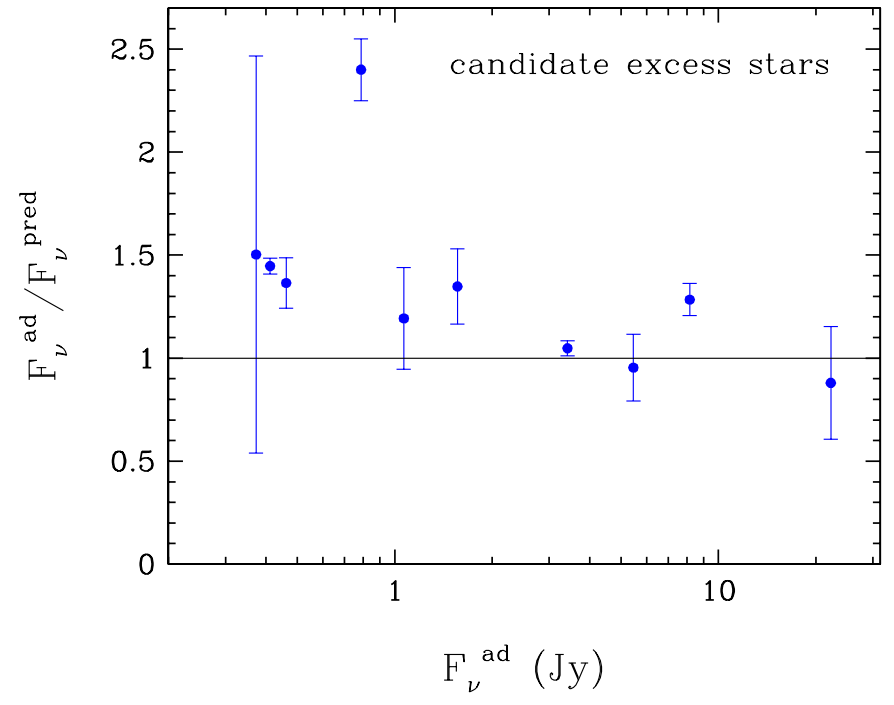

Fig. 4. The properties of the stars more than $3 \sigma$ away from the peak of the distribution presented in Fig. 3. The error bars present $\pm 3 \times \Delta F_{\nu}^{\text {ad }}$. The star HD 39060 ( $\beta$ Pic) is not included due to its off-scale positive excess of a lot.

A total of $11(=14 \%)$ targets fall outside the $\pm 3 \sigma$ interval, and 2 out of these 11 targets are below the expected flux. For these outlying stars we have plotted in Fig. 4 the ratio $F_{\nu}^{\mathrm{ad}} / F_{\nu}^{\mathrm{pred}}$. In order to indicate the significance of the deviation we have included $\pm 3 \Delta F_{\nu}^{\text {ad }}$ error bars, highlighting the uncertainties in the individual measurements.

Figure 4 indicates that 7 stars ( 6 stars plus $\beta$ Pic) with $F_{\nu}^{\text {ad }} / F_{\nu}^{\text {pred }}>0$ have fluxes which are more than $3 \Delta F_{\nu}^{\text {ad }}$ above the predicted values. These stars have been flagged in Table 1.

\subsection{Infrared excess from $K$ dwarfs}

Two of the 7 excess stars are classified as $\mathrm{K}$ dwarfs (Table 1). It is likely that Eq. (1) does not apply for these type of stars but rather the relation derived by Mathioudakis \& Doyle (1993), see also Fig. 2. Indeed, for HD 88230 Mathioudakis \& Doyle (1993) predict a photospheric flux of $513 \mathrm{mJy}$ which is above $F_{\nu}^{\text {ad }}=412 \mathrm{mJy}$ observed by us. For HD 191408 the value for $B-V$ is low, in the regime where the difference between Eq. (1) and the relation by Mathioudakis \& Doyle is small. The measured $25 \mu \mathrm{m}$ flux of $462 \mathrm{mJy}$ for HD 191408 is still more than $3 \Delta F_{\nu}^{\text {ad }}$ above the flux of 383 mJy expected for $\mathrm{K}$ dwarfs. In conclusion, we reject the detection of an excess in HD 88230.

\subsection{List of excess stars in the sample}

We have listed resulting the excess stars in Table 2. The excess emission at $25 \mu \mathrm{m}$ depends on the shape of the spectral energy distribution and the response of the filterband. We only determined the in-band excess emission for the ISO observations.
Table 2. Stars showing significant deviations from the predicted photospheric flux at $25 \mu \mathrm{m}$. The dust temperatures $T_{\mathrm{d}}$ are derived from the 25/60 flux ratio (Sect. 4.1).

\begin{tabular}{lllcc}
\hline \hline HD & \multirow{2}{*}{ name } & $\begin{array}{l}F_{\nu}^{\text {star }} \\
\text { Jy }\end{array}$ & $\begin{array}{c}\text { Excess } \\
\text { Jy }\end{array}$ & $\begin{array}{c}T_{\mathrm{d}} \\
\mathrm{K}\end{array}$ \\
\hline 38678 & $\zeta$ Lep & 0.33 & $0.54 \pm 0.05$ & $122 \pm 6$ \\
39060 & $\beta$ Pic & 0.29 & $7.06 \pm 0.26$ & $82 \pm 1$ \\
102647 & $\beta$ Leo & 1.16 & $0.39 \pm 0.07$ & $83 \pm 5$ \\
172167 & $\alpha$ Lyr & 6.35 & $2.41 \pm 0.39$ & $81 \pm 4$ \\
$191408^{*}$ & & 0.34 & $0.17 \pm 0.03$ & $(-)$ \\
216956 & $\alpha$ PsA & 3.26 & $0.20 \pm 0.07$ & $49 \pm 1$ \\
\hline
\end{tabular}

* Not a Vega-like excess star, see Sect. 4.1

If the excess emission is not point-like but comes from a region which is a significant fraction of the beam profile, then the averaging of the two flux measurements is not valid. In all positive excess cases except for HD 191408, the IRAS excess at $25 \mu \mathrm{m}$ is larger than the ISO excess. This might indicate that the excess emission is extended and has partly been resolved by ISO (cf. Fig. 5).

\section{Analysis}

\subsection{Spectral energy distributions}

Using IRAS and published ISO observations obtained at other wavelengths we have determined the far-infrared spectral energy distributions of the excess stars. The spectral energy distributions after subtraction of the photospheric emission component have been plotted in Fig. 5. When the observed emission in the IRAS $12 \mu \mathrm{m}$ band is within $5 \%$ of the estimated photospheric emission, an upper limit of $5 \%$ photospheric emission is presented.

The 12/25 flux ratio for HD 191408 yields a colour temperature of about $600 \mathrm{~K}$ assuming a $\lambda^{-1}$ dust emissivity. In combination with the ISO upper limit at $60 \mu \mathrm{m}$ we consider it more likely that the excess emission is due to coronal free-free emission. Also $F_{\nu} \propto \lambda^{-1.7}$ at $12-25 \mu \mathrm{m}$, a typical power law for free-free emission $\left(F_{\nu} \propto \lambda^{-2}-\lambda^{0}\right)$. We therefore exclude this star in the subsequent analysis.

Of the five remaining stars we have derived the 25/60 colour temperatures to analyse the temperature of the dust causing the $25 \mu \mathrm{m}$ excess emission. Assuming a $\lambda^{-1}$ dust emissivity, we find temperatures between 49 and $122 \mathrm{~K}$. The inferred temperatures are included in Table 2. HD 38678 has the highest temperature $(122 \mathrm{~K})$ and is the only star for which the $25 \mu \mathrm{m}$ excess flux density is higher than that at $60 \mu \mathrm{m}$.

\subsection{Properties of the $25 \mu \mathrm{m}$ excess stars}

The fraction of Vega-like stars in our sample with a significant excess at $25 \mu \mathrm{m}$ is 5 out of 81 or $6 \%$. Did we overlook genuine $25 \mu \mathrm{m}$ excess stars? There are two stars 


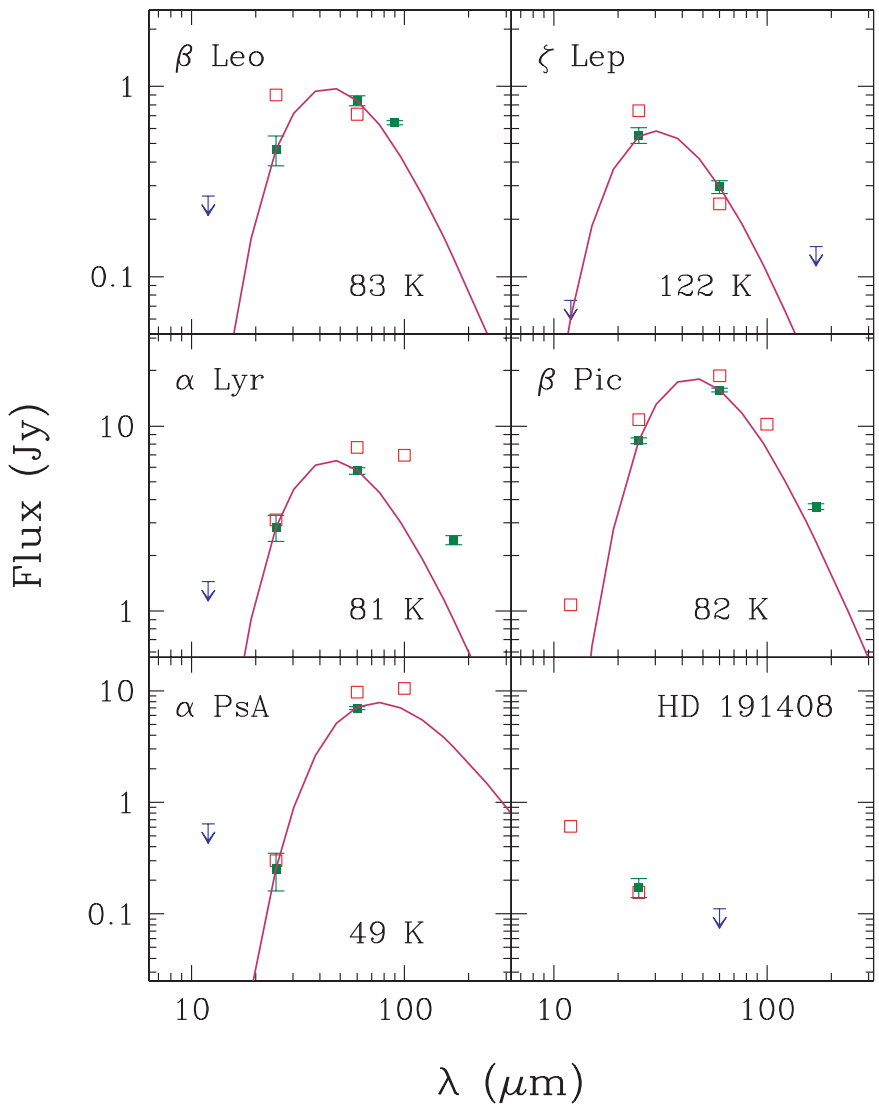

Fig. 5. The far-infrared spectral energy distributions of the $25 \mu \mathrm{m}$ excess stars (cf. Table 2) after subtraction of the photospheric emission; open squares: the excess emission derived from IRAS data, filled squares: ISO data. The ISO data at 60 and $170 \mu \mathrm{m}$ were taken from Paper I, for $\beta$ Leo we added 60 and $90 \mu \mathrm{m}$ data from the ISO archive. The solid lines are $\lambda^{-1}$ modified blackbody curves fitted to the $25 / 60$ colour of the ISO excess emission. Except for the upper limits and HD 191408, all data points were colour corrected according to the corresponding modified blackbody.

for which IRAS shows a significant excess which cannot be confirmed by ISO. The IRAS measurement of $g$ Lup (HD 139664) at $25 \mu \mathrm{m}$ (493 mJy) would indicate a strong excess above the photosphere. We find, however, an ISO flux $(253 \pm 32 \mathrm{mJy})$ which is close to the predicted photospheric flux. This star shows one of the largest discrepancies between ISO and IRAS. HD74576 (see Table 1) is the other star where IRAS would indicate an excess higher than $120 \mathrm{mJy}$ but is rejected because of an inconsistent ISO measurement. Based on these two cases we conclude that from our $25 \mu \mathrm{m}$ sample, the uncertainty in the number of excess stars is at most two, giving a most probable fraction of Vega-like stars with $25 \mu \mathrm{m}$ excess of $6 \%$ and a maximum possible fraction of $9 \%$. This is smaller than the fraction found at $60 \mu \mathrm{m}(18 \%)$ in Paper I.

\subsection{Properties of the Vega-like disks}

The non-detection of significant $25 \mu \mathrm{m}$ excess emission for all other stars in the sample shows that the Vega-like disks are generally cool: the largest fraction of the dust in the disk must be colder than $120 \mathrm{~K}$. The median $25 \mu \mathrm{m}$ flux in our sample is $F_{\nu}^{\text {median }}=310 \mathrm{mJy}([25]=3.3 \mathrm{mag})$. To be detectable in our sample the typical contrast $C_{25}$ between emission from a presumed disk and the stellar photosphere must be greater than $C_{25}>3 \sigma / F_{\nu}^{\text {median }}$, i.e. $C_{25}>0.3$. Assuming a disk temperature of $120 \mathrm{~K}$, the minimum detectable dust mass of the disk is estimated to be $M_{\mathrm{d}}=2 \times 10^{-5} M_{\oplus}$ for an A0 dwarf $\left(T_{\text {eff }}=9600 \mathrm{~K}\right)$ and $2 \times 10^{-6} M_{\oplus}$ for a G0 dwarf $\left(T_{\text {eff }}=6000 \mathrm{~K}\right)$. See Appendix A for a description of the calculation. These masses increase for lower dust temperatures. For comparison, the minimum detectable mass in the survey at $60 \mu \mathrm{m}$ is $M_{\mathrm{d}}>1 \times 10^{-5} M_{\oplus}$ (Paper I). Our Vega-like candidates are all included in the list of Paper I. Since all Vega candidates in Paper I have inferred masses larger than $1 \times 10^{-5} M_{\oplus}$ we conclude that we have detected essentially the warmest disks at $25 \mu \mathrm{m}$.

Three stars in our sample ( $\beta$ Leo, $\alpha$ Lyr, and $\beta$ Pic) show significantly more far-infrared emission at $\lambda>60 \mu \mathrm{m}$ than the modified black body energy distributions would predict, see Fig. 5. This could be an indication of the presence of colder dust material in the disk, presumably at larger radii from the stars.

The minimum detectable mass of $2 \times 10^{-6} M_{\oplus}$ for a G0 dwarf assumes an arbitrarily chosen fixed distance between the disk and the star. The detection of only A stars suggests that only stars of this stellar type are sufficiently bright to heat the dust at a minimum distance of the star. For example, a $1 \mu \mathrm{m}$ size silicate particle must be at $\sim 35$ AU from an A0 star to be at a temperature of $120 \mathrm{~K}$. At this distance, the temperature of a similar dust particle around a G0 star would be $\sim 86 \mathrm{~K}$, yielding a minimum mass of $M_{\mathrm{d}}=2 \times 10^{-5} M_{\oplus}$. It is therefore more likely that the minimum detectable mass in our sample is $M_{\mathrm{d}}=2 \times 10^{-5} M_{\oplus}$.

\section{Discussion}

The low fraction of $6 \%$ of main-sequence dwarfs exhibiting Vega-like excess emission at $25 \mu \mathrm{m}$ gives force to the result by Aumann \& Probst (1991) who carried out a similar survey at $12 \mu \mathrm{m}$. They found only 2 statistically significant excess candidates out of 548 nearby stars. These two stars ( $\beta$ Pic and $\zeta$ Lep) are also found in our sample of excess candidates. Apparently, warm debris disks are rare.

A similar study by Fajardo-Acosta et al. (2000), where 2MASS data were combined with IRAS data for a sample of 296 main-sequence stars, yielded 8 systems which have a significant excess at $12 \mu \mathrm{m}$. None of their 8 stars is in our initial sample. This low fraction $(<3 \%)$ is not inconsistent with our result at $25 \mu \mathrm{m}$. Only one of these $12 \mu \mathrm{m}$ excess stars is detected at longer wavelengths, and the spectral energy distributions of the 8 stars indicate dust temperatures in excess of $200 \mathrm{~K}$. The temperatures suggest that the systems detected by Fajardo-Acosta et al. (2000) are distinct from the systems we have detected at $25 \mu \mathrm{m}$ which all have been detected at $60 \mu \mathrm{m}$ (see Fig. 5). 
From a survey of 38 main-sequence stars using IRAS and ISOPHOT data Fajardo-Acosta et al. (1999) found no star with a significant excess at $12 \mu \mathrm{m}$, and a fraction of $\sim 14 \%$ excess stars at $20 \mu \mathrm{m}$. It is difficult to interpret this fraction since the ISOPHOT data used in their study were inconclusive, and the $20 \mu \mathrm{m}$ detections needed confirmation. In any case, the absence of $12 \mu \mathrm{m}$ detections indicates that these disks are not warmer than $200 \mathrm{~K}$.

The temperatures and the inferred upper limits for the dust emission at $25 \mu \mathrm{m}$ put strong requirements to possible ground based photometric surveys of debris disks at $20 \mu \mathrm{m}$. In order to be able to detect disks below our detection limit of $2 \times 10^{-5} M_{\oplus}$, the contrast between disk emission and photospheric emission is $<0.3$ (equivalent to larger than $1.3 \mathrm{mag}$ ). On the other hand, the accuracy of predicting the infrared photospheric flux is generally not better than $5 \%$ which limits the maximum contrast to $3.3 \mathrm{mag}$. Significant improvement can only be made by imaging the disk.

All five Vega-like candidates in our sample are young, less than 400 Myr (cf. Table 1) with spectral type A0-A3, confirming the finding by Habing et al. (1999) that debris disks are mostly found around stars that just entered the main-sequence. In fact, of the 8 stars in our sample younger than $400 \mathrm{Myr}, 5$ have a detectable dust disk at $25 \mu \mathrm{m}$, whereas none of the older stars show a significant excess.

The lower limits on the mass have been derived assuming that the size of the disk particles is much smaller than the wavelength. At $25 \mu \mathrm{m}$ this corresponds to $a \lesssim 3 \mu \mathrm{m}$, where $a$ is the radius of a grain. Larger grain sizes yield relatively lower absorption cross sections which increase our minimum mass estimate. Detailed modelling by Krügel \& Siebenmorgen (1994) and Dent et al. (2000) which includes the (observed) spatial distribution in the disk, suggests much larger grain sizes of the order of a few tens of $\mu \mathrm{m}$. Such sizes could increase our lower limit of the disk mass by one order of magnitude or more.

The dust model calculations by Li \& Greenberg (1998) for $\beta$ Pic assuming that the particles are made out of cometary material show that for a given temperature, the grains can span a whole range of distances from the star depending on the composition and mass. For $T_{\mathrm{d}}=120 \mathrm{~K}$ they find $D=20$ AU for the biggest porous silicate aggregates (of $10^{-4} \mathrm{~g}$ ) to $D=200 \mathrm{AU}$ for the smallest ones (of $10^{-14} \mathrm{~g}$ ).

\section{Conclusions}

A $25 \mu \mathrm{m}$ survey of 81 late type main-sequence dwarfs using ISO and IRAS data showed that 5 (or $6 \%$ ) of all stars in the sample exhibit significant infrared excess which can be attributed to a Vega-like dust disk. The low fraction and the fact that the disks have already been identified at $60 \mu \mathrm{m}$ indicates that the bulk emission from Vega-like disks is from cool dust $\left(T_{\mathrm{d}}<120 \mathrm{~K}\right)$.

From the detection limit of Vega-like disks we estimate a lower mass limit of $M_{\text {dust }}=2 \times 10^{-5} M_{\oplus}$ for the disks not detected by us. The survey confirms that there seems to be an absence of detectable amounts of dust at close distances $D \lesssim 20 \mathrm{AU}$ from the stars.

Acknowledgements. The ISOPHOT data presented in this paper were reduced using PIA, which is a joint development by the ESA Astrophysics Division and the ISOPHOT Consortium. We thank the referee, Dr. R. Liseau, for helpful comments leading to improvement of the manuscript.

\section{Appendix A: Derivation of mass upper limit of debris disk}

Following the treatment of Paper I, we define $C_{\nu}$, the contrast between the dust emission and the photospheric emission in the infrared:

$C_{\nu}=\frac{F_{\nu, \mathrm{dust}}}{F_{\nu, \mathrm{star}}}=\frac{L_{\nu, \mathrm{dust}}}{L_{\nu, \mathrm{star}}}$

Assuming a single sized spherical particle, the dust emission is given by:

$L_{\nu, \mathrm{dust}}=4 \pi^{2} a^{2} Q_{\nu} B_{\nu}\left(T_{\mathrm{d}}\right) N$,

where $a$ is the radius, $T_{\mathrm{d}}$ the temperature, and $Q_{\nu}$ the absorption coefficient of a dust particle. $N$ is the total number of such particles in the disk. The mass of the disk is readily computed from $M_{\text {dust }}=\frac{4}{3} \pi a^{3} N \rho_{\text {dust }}$ where $\rho_{\text {dust }}$ is the specific mass of the grain material. For dust particles smaller than the wavelength $(a<0.1 \lambda)$ one can write (e.g. Draine \& Lee 1984):

$Q=\frac{a q_{\mathrm{o}}}{\lambda^{2}}$

Approximating $L_{\text {star }} \approx A T_{\text {eff }}^{8.2}$ for main-sequence dwarfs, and assuming that the Rayleigh approximation is valid in the infrared, we obtain for the star:

$L_{\nu, \mathrm{star}}=A \frac{2 \pi k}{\sigma_{\mathrm{SB}} c^{2}} \nu^{2} T_{\mathrm{eff}}^{5.2}$

where $\sigma_{\mathrm{SB}}$ is the Stefan-Bolzmann constant. The constant $A$ can be derived from the temperature and luminosity of known stars like the Sun or Vega, we adopt $A=1.33 \times 10^{-31} L_{\odot} \mathrm{K}^{-8.2}$. Combining the above equations:

$M_{\mathrm{dust}}=A \frac{2 k}{3 \sigma} \frac{\rho_{\mathrm{dust}} T_{\mathrm{eff}}^{5.2}}{q_{\mathrm{o}} B_{\nu}\left(T_{\mathrm{d}}\right)} C_{\nu}$.

To estimate $M_{\text {dust }}$ we assume astronomical silicate (Draine \& Lee 1984) with $q_{\mathrm{o}}=1.3 \times 10^{-4} \mathrm{~m}$ and $\rho_{\text {dust }}=3.3 \mathrm{~g} \mathrm{~cm}^{-3}$. Note that this relationship is independent of the grain size $a$ as long as $a$ is significantly smaller than the infrared wavelength. 


\section{References}

Aumann, H. H., \& Probst, R. G. 1991, ApJ, 368, 264

Dent, W. R. F., Walker, H. J., Holland, W. S., \& Greaves, J. S. 2000, MNRAS, 314, 702

Draine, B. T., \& Lee, H. M. 1984, ApJ, 285, 89

Fajardo-Acosta, S. B., Beichman, C. A., \& Cutri, R. M. 2000, ApJ, 538, L155

Fajardo-Acosta, S. B., Stencel, R. E., Backman, D. E., \& Thakur, N. 1999, ApJ, 520, 215

Gabriel, C., Acosta-Pulido, J., Heinrichsen, I., Morris, H., \& Tai, W.-M. 1997, in Proceedings of the ADASS VI, ed. G. Hunt, \& H. E. Payne, ASP Conf. Ser., 125, 108

Habing, H. J., Dominik, C., Jourdain de Muizon, M., et al. 1999, Nature, 401, 456

Habing, H. J., Dominik, C., Jourdain de Muizon, M., et al. 2001, A\&A, 365, 545 (Paper I)

Jourdain de Muizon, M., Laureijs, R. J., Habing, H. J., et al. 2001, EM\&P, 85-86, 201

Kessler, M. F., Steinz, J. A., Andregg, M. E., et al. 1996, A\&A, 315, L27

Klaas, U., Krüger, H., Heinrichsen, I., Heske., A., \& Laureijs, R. 1994, ISOPHOT Observer's Manual, version 3.1

Krügel, E., \& Siebenmorgen, R. 1994, A\&A, 288, 929
Lachaume, R., Dominik, C., Lanz, T., \& Habing, H. J. 1999, A\&A, 348, 897

Laureijs, R. J., \& Jourdain de Muizon, M. 2000, Processing of ISO chopped photometry at $25 \mu \mathrm{m}$, ISO Data Centre report

Laureijs, R. J., Klaas, U., Richards, P. J., Schulz, B., \& Ábrahám, P. 2001, ISO Handbook, Volume V: PHT-The Imaging Photo-Polarimeter, ISO Data Centre, SAI-99069/Dc, Version 1.2

Leech, K., \& Pollock, A. 2000, The ISO Handbook Vol. II: ISO-The Satellite and its Data, ISO Data Centre, SAI-99082/Dc, Version 1.0

Lemke, D., Klaas, U., Abolins, J., et al. 1996, A\&A, 315, L64

Li, A., \& Greenberg, J. M. 1998, A\&A, 331, 291

Manning, V., \& Barlow, M. 1998, ApJ, 497, 330

Mathioudakis, M., \& Doyle, J. G. 1993, A\&A, 280, 181

Moshir, M. 1989, Explanatory Supplement to the IRAS Faint Source Survey (Pasadena: JPL)

Perryman, M., Lindegren, L., Kovalevsky, J., et al. 1997, A\&A, 323, L49

Plets, H. 1997, Ph.D. Thesis, Leuven University, Chapter 2

Waters, L. B. F. M., Coté, J., \& Aumann, H. H. 1987, A\&A, 172,225 